\title{
All-electron quantum Monte Carlo calculations for the noble gas atoms He to Xe
}

\author{
A. Ma, N. D. Drummond, M. D. Towler, and R. J. Needs \\ Theory of Condensed Matter Group, Cavendish Laboratory, University of Cambridge, Madingley Road, Cambridge, CB3 OHE, \\ United Kingdom \\ (Received 16 February 2005; published 21 June 2005)
}

\begin{abstract}
We report all-electron variational and diffusion quantum Monte Carlo (VMC and DMC) calculations for the noble gas atoms $\mathrm{He}, \mathrm{Ne}, \mathrm{Ar}, \mathrm{Kr}$, and $\mathrm{Xe}$. The calculations were performed using Slater-Jastrow wave functions with Hartree-Fock single-particle orbitals. The quality of both the optimized Jastrow factors and the nodal surfaces of the wave functions declines with increasing atomic number $Z$, but the DMC calculations are tractable and well behaved in all cases. We discuss the scaling of the computational cost of DMC calculations with $Z$.
\end{abstract}

DOI: 10.1103/PhysRevE.71.066704

PACS number(s): $02.70 . \mathrm{Ss}, 31.25 . \mathrm{Eb}$

\section{INTRODUCTION}

The variational quantum Monte Carlo (VMC) method and the more sophisticated diffusion quantum Monte Carlo (DMC) method [1] can yield highly accurate energies for many-electron systems. One of the main attractions of these methods is that the cost of calculating the energy of $N$ quantum particles scales roughly as $N^{2}-N^{3}$, which is better than other many-body wave function techniques. However, although the scaling with particle number is quite advantageous, the cost increases rapidly with the atomic number $Z$ of the atoms involved. Theoretical estimates of this scaling $[2,3]$ for DMC calculations have varied from $Z^{5.5}$ to $Z^{6.5}$, while a practical test [4] indicated a scaling of about $Z^{5.2}$.

Numerous all-electron DMC studies have been reported [5-17] for atoms up to $Z=10$, but very few have included heavier atoms. DMC studies of heavier atoms have normally used pseudopotentials to remove the chemically inert core electrons from the problem. However, pseudopotentials inevitably introduce some errors and it may be useful to consider how much progress can be made with all-electron DMC calculations. Accurate all-electron calculations for atoms may also be useful in constructing pseudopotentials which incorporate many-body effects. In this paper we report VMC and DMC calculations for the noble gas atoms $\mathrm{He}, \mathrm{Ne}$, $\mathrm{Ar}, \mathrm{Kr}$, and $\mathrm{Xe}$, which extends the range of atoms studied within $\mathrm{VMC}$ and DMC up to $Z=54$. The main aims of this paper are to investigate how well current all-electron DMC methods perform for heavy atoms and to study the scaling of the computational cost with $Z$.

\section{VMC AND DMC METHODS}

In the VMC method the energy is calculated as the expectation value of the Hamiltonian with an approximate manybody trial wave function containing a number of variable parameters. In the DMC method the estimate of the groundstate energy is improved by performing an evolution of the wave function in imaginary time [1]. The fermionic symmetry is maintained by the fixed-node approximation [18], in which the nodal surface of the wave function is constrained to equal that of a trial wave function. Our DMC algorithm is essentially that of Umrigar et al. [9], and we employ the modifications to the Green function for all-electron calculations proposed in that paper. All of our VMC and DMC calculations were performed using the CASINO code [19].

Our trial wave functions were of the standard SlaterJastrow form

$$
\Psi=e^{J} D_{\uparrow} D_{\downarrow} .
$$

The Jastrow factors $e^{J}$ were chosen to be functions of the variables $r_{i j}=\left|\mathbf{r}_{i}-\mathbf{r}_{j}\right|$ and $r_{i}=\left|\mathbf{r}_{i}\right|$, where $\mathbf{r}_{i}$ is the position of electron $i$ with respect to the nucleus. Our Jastrow factors [20] for $\mathrm{He}, \mathrm{Ne}, \mathrm{Ar}, \mathrm{Kr}$, and $\mathrm{Xe}$ contained a total of 26, 75, 79,80 , and 54 adjustable parameters, respectively [21]. The optimal parameter values were obtained by minimizing the variance of the energy within a VMC procedure [22,23]. The Slater determinants $D_{\sigma}$ were formed from single-particle orbitals obtained from Hartree-Fock (HF) calculations using (i) numerical integration on a radial grid and (ii) Gaussian basis sets and the CRYSTAL98 code [24]. Although the numerical orbitals are the more accurate, they are not available for molecular systems, in which Gaussian basis sets are very commonly used.

In both the VMC and DMC methods the energy is calculated as an average over a set of electron configurations of the local energy, $E_{L}=\Psi^{-1} \hat{H} \Psi$, where $\hat{H}$ is the Hamiltonian. The presence of core electrons causes two related problems. The first is that the shorter length scale variations in the wave function near a nucleus of large $Z$ require the use of a small time step. The second problem is that the fluctuations in the local energy tend to be large near the nucleus, because both the kinetic and potential energies are large. Although these fluctuations can be reduced by optimizing the trial wave function, in practice they are large for heavier atoms.

At a nucleus the exact wave function has a cusp [25] such that the divergence in the potential energy is canceled by an equal and opposite divergence in the kinetic energy. A determinant of exact HF orbitals obeys the electron-nucleus cusp condition. However, Gaussian functions are smooth, and a determinant of such orbitals cannot have a cusp, so the local energy diverges at the nucleus. In practice one finds wild oscillations in the local energy close to the nucleus, which 
TABLE I. Total energies of the noble gas atoms and the percentages of the correlation energies $E_{\mathrm{c}}$ retrieved. $(\mathrm{G})$ denotes a calculation with a Gaussian basis set and $(\mathrm{N})$ denotes numerical orbitals. The "exact" energies were obtained from data in the indicated references.

\begin{tabular}{|c|c|c|c|c|}
\hline Atom & Method & $\begin{array}{l}\text { Orb. } \\
\text { type }\end{array}$ & $\begin{array}{l}\text { Total energy } \\
\text { (a.u.) }\end{array}$ & $E_{\mathrm{c}}$ \\
\hline \multirow{8}{*}{$\mathrm{He}$} & $\mathrm{HF}$ & $\mathrm{G}$ & -2.86165214 & $0 \%$ \\
\hline & $\mathrm{HF}$ & $\mathrm{N}$ & -2.86168000 & $0 \%$ \\
\hline & VMC & $\mathrm{G}$ & $-2.903499(8)$ & $99.5 \%$ \\
\hline & VMC & $\mathrm{N}$ & $-2.903527(9)$ & $99.5 \%$ \\
\hline & $\mathrm{DMC}$ & $\mathrm{G}$ & $-2.903732(5)$ & $100 \%$ \\
\hline & DMC & $\mathrm{N}$ & $-2.903719(2)$ & $100 \%$ \\
\hline & "Exact" [27] & - & -2.903724 & $100 \%$ \\
\hline & $\mathrm{HF}$ & $\mathrm{G}$ & -128.53832860 & $0 \%$ \\
\hline \multirow{5}{*}{$\mathrm{Ne}$} & $\mathrm{HF}$ & $\mathrm{N}$ & -128.54709811 & $0 \%$ \\
\hline & VMC & G & $-128.8794(4)$ & $85 \%$ \\
\hline & VMC & $\mathrm{N}$ & $-128.891(5)$ & $88 \%$ \\
\hline & DMC & G & $-128.9232(5)$ & $96 \%$ \\
\hline & DMC & $\mathrm{N}$ & $-128.9231(1)$ & $96 \%$ \\
\hline \multirow{5}{*}{$\mathrm{Ar}$} & “Exact” [28] & - & -128.939 & $100 \%$ \\
\hline & $\mathrm{HF}$ & $\mathrm{N}$ & -526.81751277 & $0 \%$ \\
\hline & VMC & $\mathrm{N}$ & $-527.3817(2)$ & $77 \%$ \\
\hline & DMC & $\mathrm{N}$ & $-527.4840(2)$ & $91 \%$ \\
\hline & "Exact" [29] & - & -527.55 & $100 \%$ \\
\hline \multirow{3}{*}{$\mathrm{Kr}$} & $\mathrm{HF}$ & $\mathrm{N}$ & -2752.05497715 & $0 \%$ \\
\hline & VMC & $\mathrm{N}$ & $-2753.2436(6)$ & $57 \%$ \\
\hline & DMC & $\mathrm{N}$ & $-2753.7427(6)$ & $82 \%$ \\
\hline \multirow{5}{*}{$\mathrm{Xe}$} & "Exact" [30] & - & -2754.13 & $100 \%$ \\
\hline & $\mathrm{HF}$ & $\mathrm{N}$ & -7232.13836331 & $0 \%$ \\
\hline & VMC & $\mathrm{N}$ & $-7233.700(2)$ & $46 \%$ \\
\hline & DMC & $\mathrm{N}$ & $-7234.785(1)$ & $77 \%$ \\
\hline & "Exact" [30] & - & -7235.57 & $100 \%$ \\
\hline
\end{tabular}

increase the variance of the energy in VMC calculations and lead to time step errors and even numerical instabilities in DMC calculations. To solve this problem we make small corrections to the single-particle orbitals close to the nucleus, which impose the correct cusp behavior [26].

\section{RESULTS}

\section{A. Quality of the trial wave functions}

The main results of our HF, VMC, and DMC calculations are shown in Table I. Our HF energy for He with Gaussian orbitals is very close to the result with the "exact" numerical orbitals, indicating the high quality of the Gaussian basis set used. For Ne the HF energy with Gaussian orbitals is a little higher than the value with the numerical orbitals, although this difference is not large enough to affect the DMC results. We experimented with various Gaussian basis sets for the heavier noble gas atoms and found that the basis set errors at the HF level tend to increase significantly with atomic num- ber. In the case of Xe our best Gaussian basis set gave an error of 0.11 a.u. For Ar, Kr, and Xe we therefore used only the numerical orbitals.

The exact ground-state wave function of a two-electron atom is a nodeless function of $r_{1}, r_{2}$, and $r_{12}$, which is the same form as our trial wave function for He. We therefore expect to obtain a highly accurate trial wave function for $\mathrm{He}$. We refer to the difference in the HF and DMC energies as the "DMC correlation energy." If one keeps the orbitals fixed and varies the Jastrow factor, then the lowest energy one could obtain is the DMC energy. The percentage of the DMC correlation energy retrieved at the VMC level is therefore a measure of the quality of the Jastrow factor. From the data in Table I we find that our VMC calculations retrieve $99.5 \%$, $91 \%, 85 \%, 70 \%$, and $59 \%$ of the DMC correlation energy for $\mathrm{He}, \mathrm{Ne}, \mathrm{Ar}, \mathrm{Kr}$, and $\mathrm{Xe}$, respectively. We believe that the decrease in the quality of the Jastrow factor with increasing $Z$ arises from the increasing inhomogeneity of the atoms. Creating accurate Jastrow factors for all-electron studies of heavy atoms is a challenging problem.

Our VMC and DMC energies for $\mathrm{Ne}$ obtained with the numerical orbitals are very close to those obtained in our earlier work [20]. Huang et al. [13] obtained a VMC energy of $-128.9008(1)$ a.u., which is only slightly lower than our value, although they also optimized the orbitals. Our DMC energies for $\mathrm{Ne}$ are within error bars of those reported by Umrigar et al. [9], but the remaining fixed-node error of 0.016 a.u. is substantial.

From the data in Table I we observe that the percentages of the correlation energy missing at the DMC level are $0 \%$, $4 \%, 9 \%, 18 \%$ and $23 \%$ for $\mathrm{He}, \mathrm{Ne}, \mathrm{Ar}, \mathrm{Kr}$ and $\mathrm{Xe}$, respectively. This indicates that the size of the fixed-node error increases rapidly with $Z$.

\section{B. Theoretical scaling with atomic number}

It is of interest to study the CPU time required to obtain a fixed standard error in the mean energy, $\Delta$, as a function of the atomic number $Z$. The required CPU time $T$ can be written as

$$
T \propto M T_{C},
$$

where $M$ is the total number of generations of electron configurations and $T_{C}$ is the CPU time for one move of $C$ configurations, where $C$ is the average number of configurations in a generation.

Ceperley [3] showed that $\Delta^{2}$ can be written as the sum of two terms; the first corresponds to the square of the standard error evaluated as if the DMC energies were uncorrelated, and the second accounts for the effects of correlations. In DMC calculations the time step $\tau$ is normally chosen to be small, and the correlations between configurations at successive generations are large, so that the second of these terms dominates. Ceperley showed that this term is given approximately by [3] 


$$
\Delta^{2}=\frac{2\left|E_{\mathrm{VMC}}-E_{\mathrm{DMC}}\right|}{\tau M C} .
$$

Since $\Delta^{2}$ is inversely proportional to the total number of configurations, we obtain

$$
T \propto \tau^{-1}\left|E_{\mathrm{VMC}}-E_{\mathrm{DMC}}\right| T_{C} .
$$

Ceperley used the simple approximation $\left|E_{\mathrm{VMC}}-E_{\mathrm{DMC}}\right|$ $\propto E_{\mathrm{c}}$ and the approximate scaling $E_{\mathrm{c}} \propto Z^{1.5}$. He also argued that avoiding large time step errors requires $\tau \propto Z^{-2}$, as the average distance diffused should be smaller than the size of the $1 s$ orbital, which is proportional to $Z^{-1}$. Finally, he used $T_{C} \propto Z^{2}$ to obtain an overall scaling of

$$
T \propto Z^{5.5} .
$$

Hammond et al. [2] argued along similar lines, although they chose $T_{C} \propto Z^{3}$, leading to an overall scaling of $T \propto Z^{6.5}$. In what follows we examine some aspects of these arguments.

\section{Numerical tests of scaling with atomic number}

Discussions of the actual scaling of the computational cost of calculations with system size or atomic number are fraught with difficulties. The results depend on the computers on which the calculations are run, the algorithms used, and the details of the software used. Our calculations are run on parallel computers in which each processor deals with a small number of electronic configurations (one in the VMC method and roughly ten in the DMC method). The interprocessor communications are negligible in the VMC method and small in the DMC method, and the computational cost is inversely proportional to the number of processors used. All of the DMC results used for determining the scaling of the computational cost with atomic number were performed on 96 processors of a Sunfire Galaxy machine, although most of the variance minimizations were performed on a cluster of 16 xeon dual processors.

To ensure that time step errors are small the DMC time step should be chosen so that the probability of a move being accepted is high. For the DMC results reported in Table I we used time steps of $0.02,0.0025,0.0009,0.00035$, and 0.0002 a.u. for $\mathrm{He}, \mathrm{Ne}, \mathrm{Ar}, \mathrm{Kr}$, and $\mathrm{Xe}$, respectively, which were chosen so that in each case slightly more than $99 \%$ of the proposed moves were accepted. These time steps scale as $Z^{-1.41}$, which is significantly weaker than the $Z^{-2}$ scaling used in the earlier theoretical estimates [2,3]. We therefore expect the time step bias in our DMC results to increase with $Z$.

We thoroughly investigated the time step dependence of the energies for $\mathrm{He}$ and $\mathrm{Ne}$, concluding that they are negligible compared with the statistical error bars given in Table I. For each of $\mathrm{Ar}, \mathrm{Kr}$, and $\mathrm{Xe}$ we performed calculations at four different time steps, and we estimate that the time step errors in the corresponding DMC energies are less than 0.002 a.u. (Ar), 0.01 a.u. (Kr), and 0.015 a.u. (Xe). It is likely that the larger time step errors in our DMC results for the heavier atoms arise both from the reduction in the quality of the trial wave functions and the poorer sampling of the core electrons.

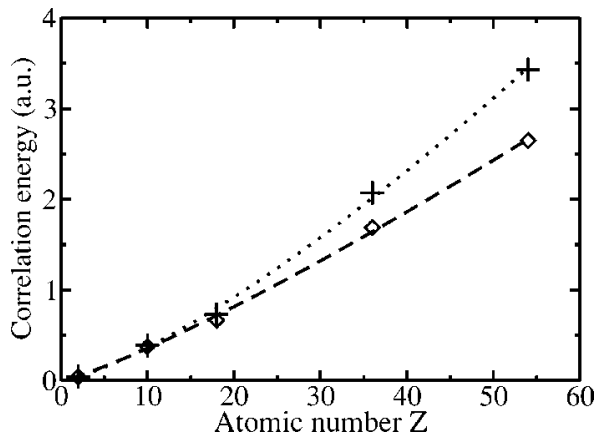

FIG. 1. The correlation energy $E_{\mathrm{c}}$ as a function of atomic number $Z$. Crosses: "exact" values. Diamonds: DMC values. The dotted line is a fit to the "exact" values giving $E_{\mathrm{c}} \propto Z^{1.33}$, while the dashed line is a fit to the DMC data giving $E_{\mathrm{c}} \propto Z^{1.26}$.

The correlation energy is normally defined as the difference between the exact nonrelativistic ground-state energy and the HF energy, assuming static point nuclei. Accurate estimates of the correlation energies of neutral atoms for $Z$ $=2-18$ are given by Chakravorty and Davidson [29], while Clementi and Hofmann [30] give values for $\mathrm{Kr}$ and $\mathrm{Xe}$ which, while probably not as accurate as those for the lighter atoms, are expected to be quite reliable. We will take these as our reference data and refer to them as the "exact" correlation energies and, when added to the Hartree-Fock energies, the "exact" energies.

Figure 1 shows the correlation energy as a function of $Z$ from our DMC data and the estimates of Chakravorty and Davidson [29] $(Z=2-18)$ and Clementi and Hofmann [30] $(Z=36,54)$. It is clear that (apart from He) DMC underestimates the correlation energy and that the underestimation becomes more severe at larger $Z$. The best power-law fit to the "exact" data for the noble gas atoms gives $E_{\mathrm{c}} \propto Z^{1.33}$, while for our DMC data we obtain $Z^{1.26}$. The scaling of $Z^{1.5}$ assumed in the earlier theoretical estimates $[2,3]$ is somewhat of an overestimate.

As mentioned in Sec. III B, the quantity which actually enters Ceperley's approximation of Eq. (3) for the variance of the DMC energy is the difference between the variational and $\mathrm{DMC}$ energies, $\left|E_{\mathrm{VMC}}-E_{\mathrm{DMC}}\right|$. Using the $\mathrm{VMC}$ and $\mathrm{DMC}$ data given in Table I we find $\left|E_{\mathrm{VMC}}-E_{\mathrm{DMC}}\right| \propto Z^{2.62}$. The reason that this quantity increases more rapidly with $Z$ than $E_{\mathrm{c}}$ is that the percentage of the correlation energy retrieved in our VMC calculations decreases with $Z$ more rapidly than in our DMC calculations.

We can also test Eq. (3) directly by comparing the difference between the variational and DMC energies $\mid E_{\mathrm{VMC}}$

TABLE II. The quantity $1 / 2 \tau M C \Delta^{2}$ and the difference between the VMC and DMC energies.

\begin{tabular}{ccc}
\hline \hline Atom & $1 / 2 \tau M C \Delta^{2}$ (a.u.) & $\left|E_{\mathrm{VMC}}-E_{\mathrm{DMC}}\right|$ (a.u.) \\
\hline $\mathrm{He}$ & 0.00019 & 0.00019 \\
$\mathrm{Ne}$ & 0.024 & 0.032 \\
$\mathrm{Ar}$ & 0.11 & 0.10 \\
$\mathrm{Kr}$ & 0.66 & 0.50 \\
$\mathrm{Xe}$ & 1.3 & 1.0 \\
\hline \hline
\end{tabular}




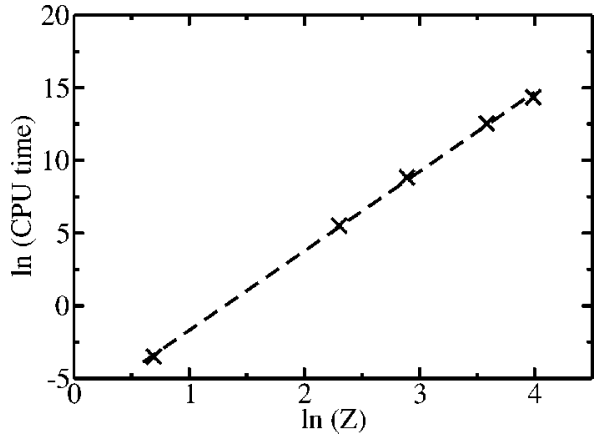

FIG. 2. The logarithm of the CPU time required to obtain a fixed error bar in the energy versus $\ln (Z)$ for our DMC calculations. The dashed line shows the fitted scaling of $Z^{5.47}$. The CPU times are measured in seconds.

$-E_{\mathrm{DMC}} \mid$ with the quantity $\frac{1}{2} \tau M C \Delta^{2}$. Serial correlation of the data has been taken into account when computing the variance over the run. The results shown in Table II indicate that the two quantities are in good agreement, which is rather satisfactory considering the large range of $Z$ and the very different qualities of trial wave functions used. The quantity $\frac{1}{2} \tau M C \Delta^{2}$ is fitted by a scaling of $Z^{2.71}$.

We found that the computational cost of moving all the electrons in a configuration scaled as $Z^{1.35}$ in our DMC calculations. This is rather better than the scalings assumed by Ceperley [3] $\left(Z^{2}\right)$ and by Hammond et al. [2] $\left(Z^{3}\right)$. If we studied a system containing many atoms, the scaling of the computational cost for moving all the electrons in a configuration would be expected to increase roughly as $N^{2}$, although the use of localized Wannier functions could reduce this to $N$ [31]. find

Putting together our scalings for the factors in Eq. (4) we

$$
T \propto Z^{1.41} \times Z^{2.62} \times Z^{1.35}=Z^{5.38} .
$$

We can now compare this with the actual DMC computations reported in Table I. In Fig. 2 we show the logarithm of the CPU time as a function of $Z$ for a given standard error of the mean. The best fit gives a scaling of $Z^{5.47}$, in good agree- ment with the prediction of $Z^{5.38}$ from Eq. (4).

As mentioned before, our DMC results for the heavier atoms suffer from significant timestep errors. If we adopt the $Z^{-2}$ scaling for the time step instead of the $Z^{-1.41}$ used above, we obtain an overall scaling of $T \propto Z^{5.97}$, which is higher than the value of $T \propto Z^{5.2}$ obtained in the practical tests of Hammond et al. [4]. Moreover, it seems likely that an even more rapid scaling would be required to achieve a time step error independent of $Z$.

\section{CONCLUSIONS}

We have applied the VMC and DMC methods to noble gas atoms up to $\mathrm{Xe}(Z=54)$, using Slater-Jastrow wave functions with Hartree-Fock single-particle orbitals. The percentage of the DMC correlation energy obtained at the VMC level decreases with $Z$, indicating that the quality of our Jastrow factors decreases with $Z$. The percentage of the exact correlation energy retrieved at the DMC level also decreases with $Z$, indicating that the quality of the HF nodal surface deteriorates with increasing $Z$.

Our study shows that Ceperley's expression [3] for the variance of the DMC energy [Eq. (3)] is accurate to better than a factor of 2 for the systems studied here. The computational cost required to obtain a fixed statistical error bar in the energy scaled as $Z^{5.47}$, but in these calculations the time step error increased significantly with $Z$. The scaling required to achieve a time step error independent of $Z$ is difficult to estimate, but it would certainly be higher than $Z^{5.47}$. However, it may well be reasonable to incur substantial time step errors deep in the core of the atom when we calculate chemical properties which are related to the valence electrons.

\section{ACKNOWLEDGMENTS}

We thank John Trail for providing the numerical HartreeFock atomic orbitals and energies. We acknowledge financial support from the Engineering and Physical Sciences Research Council of the United Kingdom. Access to the Sunfire Galaxy computer was provided by the Cambridge-Cranfield High Performance Computing Facility.
[1] W. M. C. Foulkes, L. Mitas, R. J. Needs, and G. Rajagopal, Rev. Mod. Phys. 73, 33 (2001).

[2] B. L. Hammond, P. J. Reynolds, and W. A. Lester, Jr., J. Chem. Phys. 87, 1130 (1987).

[3] D. M. Ceperley, J. Stat. Phys. 43, 815 (1986).

[4] Data from B. L. Hammond, as reported in D. M. Ceperley and L. Mitas, Adv. Chem. Phys. 93, 1 (1996).

[5] P. J. Reynolds, D. M. Ceperley, B. J. Alder, and W. A. Lester, Jr., J. Chem. Phys. 77, 5593 (1982).

[6] P. J. Reynolds, R. N. Barnett, B. L. Hammond, and W. A. Lester, J. Stat. Phys. 43, 1017 (1986).

[7] J. Vrbik, M. F. DePasquale, and S. M. Rothstein, J. Chem. Phys. 88, 3784 (1988).
[8] W. A. Lester, Jr. and B. L. Hammond, Annu. Rev. Phys. Chem. 41, 283 (1990).

[9] C. J. Umrigar, M. P. Nightingale, and K. J. Runge, J. Chem. Phys. 99, 2865 (1993).

[10] S. D. Kenny, G. Rajagopal, and R. J. Needs, Phys. Rev. A 51, 1898 (1995).

[11] A. Lüchow and J. B. Anderson, J. Chem. Phys. 105, 7573 (1996).

[12] T. Yoshida and G. Miyako, J. Chem. Phys. 107, 3864 (1997).

[13] C. J. Huang, C. J. Umrigar, and M. P. Nightingale, J. Chem. Phys. 107, 3007 (1997).

[14] Y. Shlyakhter, S. Sokolova, A. Lüchow, and J. B. Anderson, J. Chem. Phys. 110, 10725 (1999). 
[15] A. Sarsa, J. Boronat, and J. Casulleras, J. Chem. Phys. 116, 5956 (2002).

[16] M. Casula and S. Sorella, J. Chem. Phys. 119, 6500 (2003).

[17] M. Casula, C. Attaccalite, and S. Sorella, J. Chem. Phys. 121, 7110 (2004).

[18] J. B. Anderson, J. Chem. Phys. 65, 4121 (1976).

[19] R. J. Needs, M. D. Towler, N. D. Drummond, and P. R. C. Kent, CASINO, version 1.7 User Manual, University of Cambridge, Cambridge, 2003.

[20] N. D. Drummond, M. D. Towler, and R. J. Needs, Phys. Rev. B 70, 235119 (2004).

[21] For Xe, VMC energies of $-7233.700(2)$ a.u. and -7233.713(4) a.u. were obtained using Jastrow factors with 54 and 80 parameters, respectively. Since the lowering of the energy due to the use of a larger number of parameters was small, the 54-parameter Jastrow factor was used in the DMC calculations.

[22] C. J. Umrigar, K. G. Wilson, and J. W. Wilkins, Phys. Rev. Lett. 60, 1719 (1988).
[23] P. R. C. Kent, R. J. Needs, and G. Rajagopal, Phys. Rev. B 59, 12344 (1999).

[24] V. R. Saunders, R. Dovesi, C. Roetti, M. Causà, N. M. Harrison, R. Orlando, and C. M. Zicovich-Wilson, CRYSTAL98, User's Manual, University of Torino, Torino, 1998.

[25] T. Kato, Commun. Pure Appl. Math. 10, 151 (1957).

[26] A. Ma, M. D. Towler, N. D. Drummond, and R. J. Needs (unpublished).

[27] C. L. Pekeris, Phys. Rev. 112, 1649 (1958).

[28] E. R. Davidson, S. A. Hagstrom, S. J. Chakravorty, V. M. Umar, and C. F. Fischer, Phys. Rev. A 44, 7071 (1991); S. J. Chakravorty, S. R. Gwaltney, E. R. Davidson, F. A. Parpia, and C. F. Fischer, ibid. 47, 3649 (1993).

[29] S. J. Chakravorty and E. R. Davidson, J. Phys. Chem. 100, 6167 (1996).

[30] E. Clementi and D. W. M. Hofmann, THEOCHEM 330, 17 (1995).

[31] A. J. Williamson, R. Q. Hood, and J. C. Grossman, Phys. Rev. Lett. 87, 246406 (2001). 